\title{
Shear buckling resistance of non-uniform thickness bridge girder webs
}

\section{Mikael Möller \\ $\mathrm{PhD}$}

Areva NP Uddcomb

Helsingborg, Sweden

mikael.moller@areva.com

Mikael Möller, born 1965, works with structural assessments of nuclear power plant components at Areva NP. He is a member of the Areva NP Expert community.

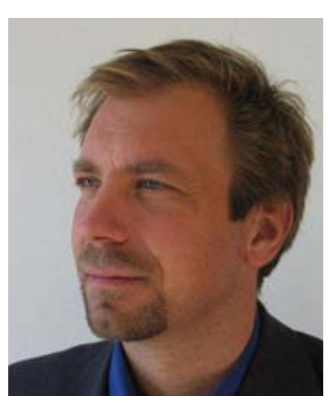

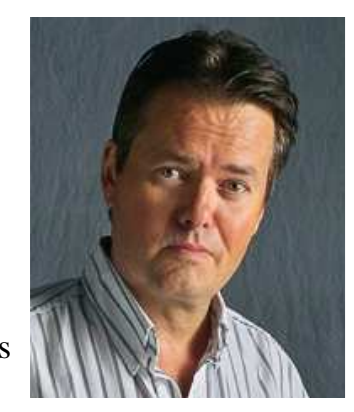
\author{
professor in Composite Structures \\ at Luleå University. He holds a \\ position as Bridge Market \\ Manager at Ramboll Sweden. \\ Peter Collin \\ Professor, PhD \\ Ramböll Consultants \& \\ Luleå University \\ Luleå, Sweden \\ peter.collin@ramboll.se \\ Peter Collin, born 1960, is
}

\section{Summary}

In very large steel bridge girders, the web often must be composed of more than one plate strip. As an alternative to longitudinal stiffeners of a slender web of uniform thickness, the bottom web plate strip my be designed as a vertical extension of the bottom flange - thicker than the upper web strip. A thicker bottom web strip enhances both the shear buckling resistance of the web and the bending moment resistance of the cross-section. The magnitude of these beneficial effects are adressed in this article. Moreover, the effect on the shear-bending resistance interaction is investigated. Nonlinear finite element simulation is conducted and comparison to the predictions of the EC 3 is made.

Keywords: Bridge girders, shear buckling, shear-bending interaction, non-linear finite element simulation.

\section{Introduction}

In Swedish steel and composite bridges the post-critical behaviour of the web plates has been accounted for since some 20 years, and for other kinds of steel structures for 40 years. This means that the flanges can be fully utilized in bending, although this is not the case with the slender web plates connecting them. In other European countries this is not allowed, and this means that the web often have longitudinal stiffeners, which increase the critical stress for the web in bending, but also the total costs. Another aspect is that the web must be able to carry the vertical shear force, and this can lead to demands for either thicker webs or longitudinal stiffeners to increase the buckling coefficient, $k_{\tau}$. Since the shear capacity increases much faster than the thickness of the web, it is however often advantageous to increase the web a few mm's, instead of introducing longitudinal stiffeners.

For very high girders, however, the web must often be composed of more than one plate strip. This gives an excellent opportunity to increase the thickness of the web part in compression, which will increase the shear capacity, and even more so the bending capacity of the steel cross section. If for example the lower $25 \%$ of a cross section at support is composed of a thicker plate, the bending stresses have decreased to $50 \%$ at the intersection to the thinner web plate. Furthermore, most of the extra material placed in the web can be excluded from the bottom flange. Although the material put in the flange has a somehow better cantilever arm in bending, the shear lag in a wide bottom flange to some extent can compensate for this.

\section{Shear buckling of uniform thickness slender web plates}

\subsection{Resistance prediction of the EC3}

The shear buckling resistance of an un-stiffened slender uniform thickness web is governed by the EC3 slenderness parameter, [1],

$$
\bar{\lambda}_{w}=\sqrt{\frac{\tau_{y}}{\tau_{c r}}}=\sqrt{\frac{f_{y}}{\sqrt{3} \tau_{c r}}}=0,76 \sqrt{\frac{f_{y}}{\tau_{c r}}}
$$


For a long web this turns into

$$
\bar{\lambda}_{w \infty}=0,35 \frac{h_{w}}{t} \sqrt{\frac{f_{y}}{E}}
$$

For a rectangular web by which the length to width ratio is $a / h_{w}$ it holds

$$
\bar{\lambda}_{w}=\frac{0,81}{\sqrt{5,34+4\left(h_{w} / a\right)^{2}}} \frac{h_{w}}{t} \sqrt{\frac{f_{y}}{E}}
$$

In any case, the buckling reduction factor for a slender web with rigid end post is given by

$$
\chi_{w}=\frac{1,37}{0,7+\bar{\lambda}_{w}}
$$

and the resistance is

$$
V_{R}=\frac{\chi_{w} f_{y} h_{w} t}{\sqrt{3}}
$$

Considering a numerical example by which $h_{w}=4 \mathrm{~m}, a=8 \mathrm{~m}, t=20 \mathrm{~mm} \&$ steel $S 355$ - which would correspond to a very slender large bridge girder web with c/c cross-beams $8 \mathrm{~m}-$ yields $\bar{\lambda}_{w}=2,65, \chi_{w}=0,40$ and the shear resistance $V_{R}=6600 \mathrm{kN}$.

\subsection{Resistance prediction of nonlinear finite element simulations}

The shear buckling resistance is simulated by means of non-linear finite element analysis taking into account material plasticity as well as initial geometrical imperfections. The model is shown in figure 1 below and shares the same geometrical parameters as defined above.

Residual stresses are not included explicitly but are assumed covered by the magnitude of the geometrical imperfections. The geometrical initial imperfections are taken affine to the first eigenmode in accordance with figure 1 below. The amplitude of the initial buckles is taken as $h_{w} / 200=20 \mathrm{~mm}$.
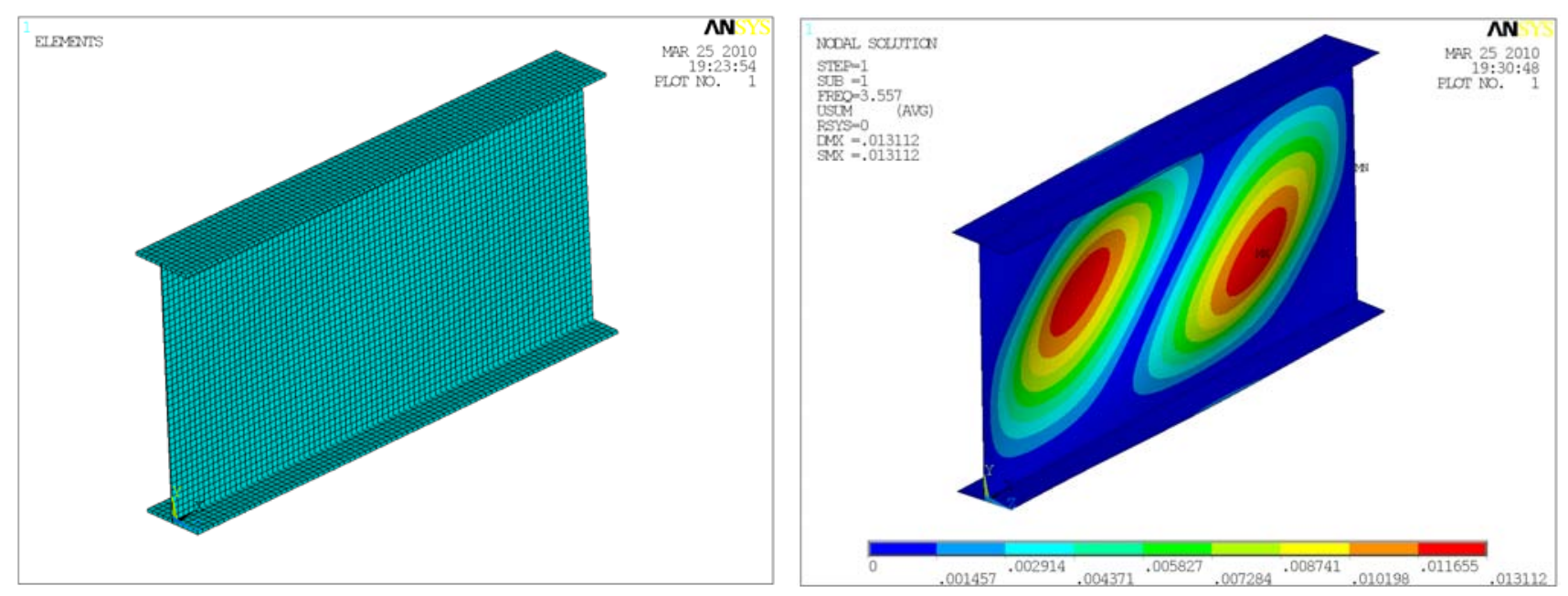

Fig. 1: Finite element model with four-noded shells (left). Assumed initial deformations affine with first eigenmode (right).

The resistance from the nonlinear simulation as defined above is about $6900 \mathrm{kN}$. The state of deformation and plastic strains is shown in figure 2 below. In the figure, the contour limit for plastic strains is taken low so as reveal the extent of the plastic regions. The strains in the figure are membrane strains - hence plate bending strains are excluded. 


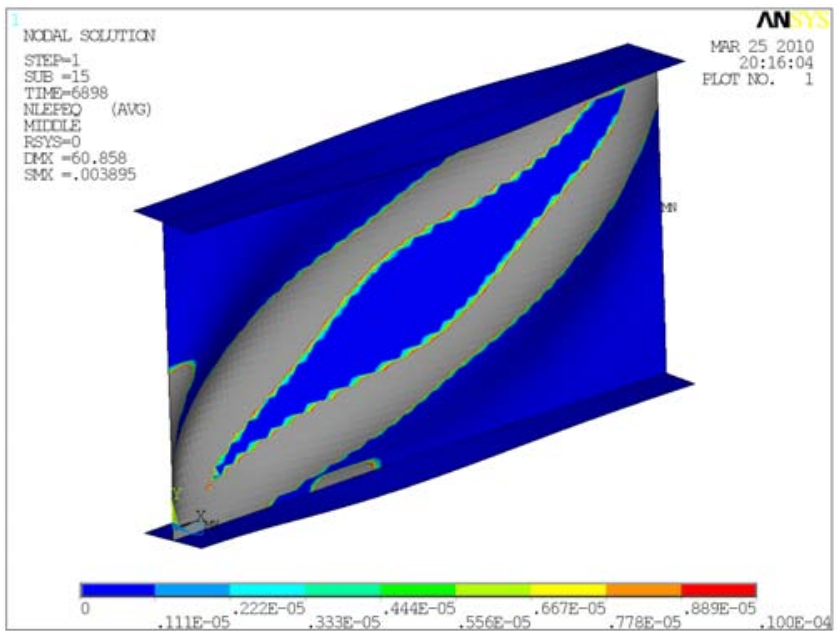

Fig. 2: Resistance $6900 \mathrm{kN}$ from nonlinear analysis.
Obviously the magnitude of initial imperfections is somewhat arbitrary although quite reasonable. The resulting resistance of $6900 \mathrm{kN}$ deviates by some $5 \%$ to the prediction $6600 \mathrm{kN}$ by the EC 3 above. This is clearly reasonable enough.

The objective of this simulation was to demonstrate the ability to simulate shear buckling and to predict the resistance with adequate precision.

\section{Shear buckling of non-uniform thickness slender web plates}

Analysis is conducted for bottom portions of the web being $50 \mathrm{~mm}$ thick - which might be seen as an extension of the bottom flange. The portions investigated are $500 \mathrm{~mm}, 1000 \mathrm{~mm} \& 1500 \mathrm{~mm}$. For each configuration, a critical load analysis is conducted which allows for a code evaluation of the shear resistance. In addition to that, complete nonlinear analysis is also conducted so as to compare the resistances.

For the case of the bottom $500 \mathrm{~mm}$ of the web being $50 \mathrm{~mm}$ thick, the critical load is $4250 \mathrm{kN}$ in accordance with figure 3 below. The resistance as determined by nonlinear analysis is $7650 \mathrm{kN}$. Using the critical load for subsequent code evaluation it is obtained

$$
\begin{aligned}
& \bar{\lambda}_{w}=\sqrt{\frac{V_{y}}{V_{c r}}}=\sqrt{\frac{0,355 \cdot(3500 \cdot 20+500 \cdot 50)}{\sqrt{3} \cdot 4250}}=2,14 \\
& \chi_{w}=\frac{1,37}{0,7+2,14}=0,48 \\
& V_{R}=\frac{0,48 \cdot(3500 \cdot 20+500 \cdot 50) \cdot 0,355}{\sqrt{3}}=9300 \mathrm{kN}
\end{aligned}
$$

This resistance is obviously an over-estimation. Using a fictitious web area for the last step in analysis - corresponding to $4000 \times 20 \mathrm{web}$ - results in a resistance

$$
V_{R}=\frac{0,48 \cdot 4000 \cdot 20 \cdot 0,355}{\sqrt{3}}=7880 \mathrm{kN}
$$

which is differs only $3 \%$ from the resistance predicted by the nonlinear analysis. Increasing the bottom part of the web to $1000 \mathrm{~mm}$ yields a critical shear load of $5200 \mathrm{kN}$ as shown if figure 4 below. Using the same analysis route as above - i.e. accounting for the enhanced critical load but considering a web area of $4000 \times 20$ - yields $\bar{\lambda}_{w}=1,94, \chi_{w}=0,52$ and a resistance of $V_{R}=8520 \mathrm{kN}$. As seen in figure 4 below, the resistance from nonlinear analysis is $8540 \mathrm{kN}$ and so the difference is less than $1 \%$.

Increasing the bottom part further to $1500 \mathrm{~mm}$, the critical load is magnified to a $6740 \mathrm{kN}$ as shown in figure 5. This yields $\bar{\lambda}_{w}=1,70, \chi_{w}=0,57$ and a resistance of $V_{R}=9360 \mathrm{kN}$. As seen in figure 5 below, the resistance from nonlinear analysis is $9760 \mathrm{kN}$ and so the difference is about $4 \%$. 

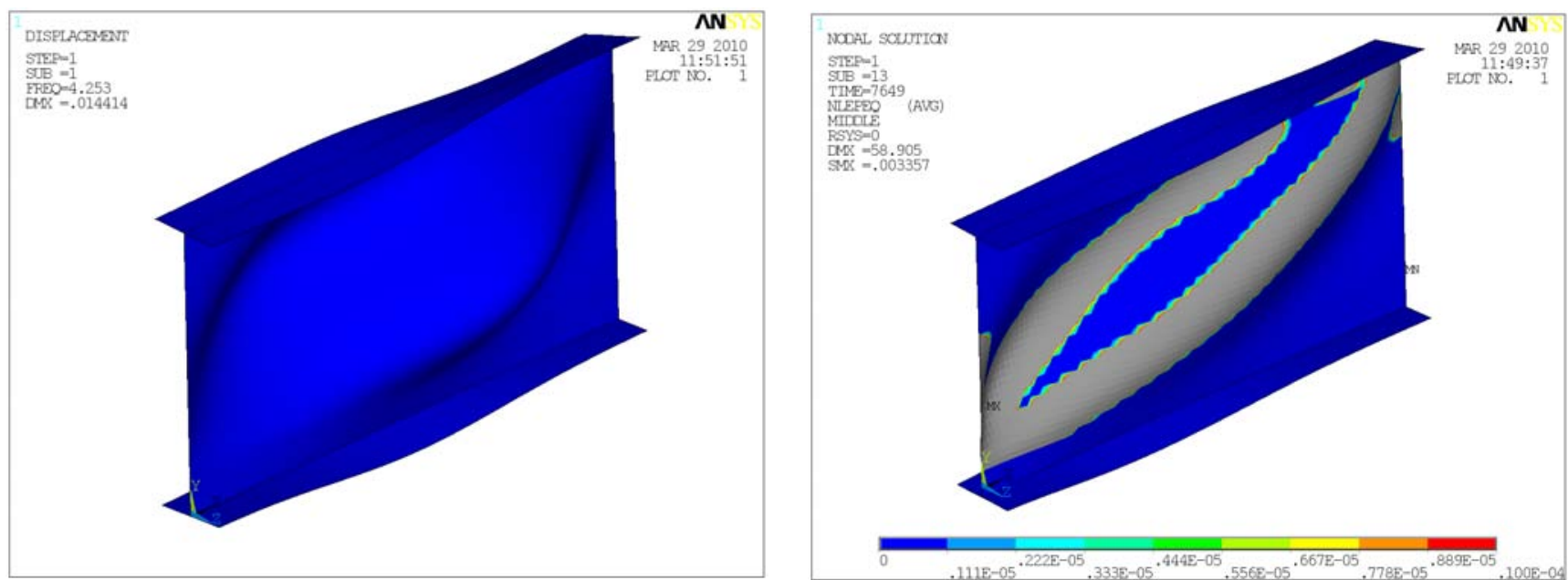

Fig. 3: Bottom $500 \mathrm{~mm}$ of web with thickness $50 \mathrm{~mm}$. Critical shear load $4250 \mathrm{kN}$ (left). Resistance $7650 \mathrm{kN}$ as determined by nonlinear analysis.
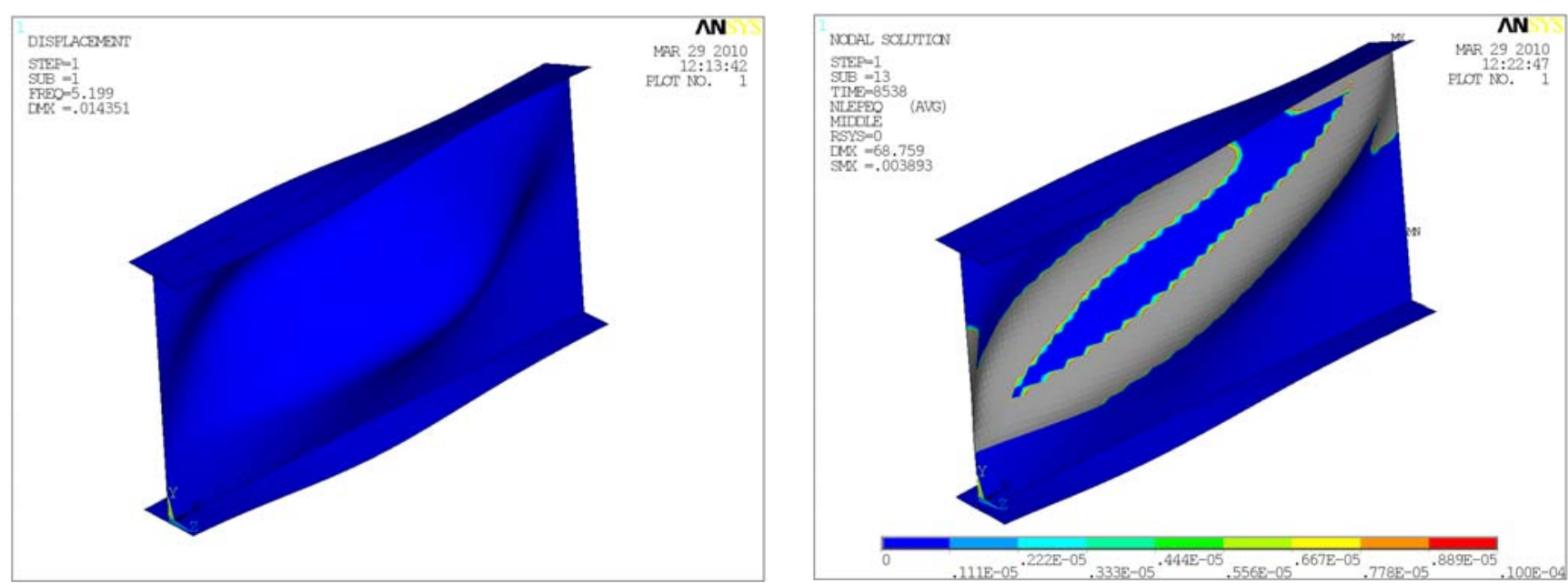

Fig. 4: Bottom $1000 \mathrm{~mm}$ of web with thickness $50 \mathrm{~mm}$. Critical shear load $5200 \mathrm{kN}$ (left). Resistance 8540 $k N$ as determined by nonlinear analysis.
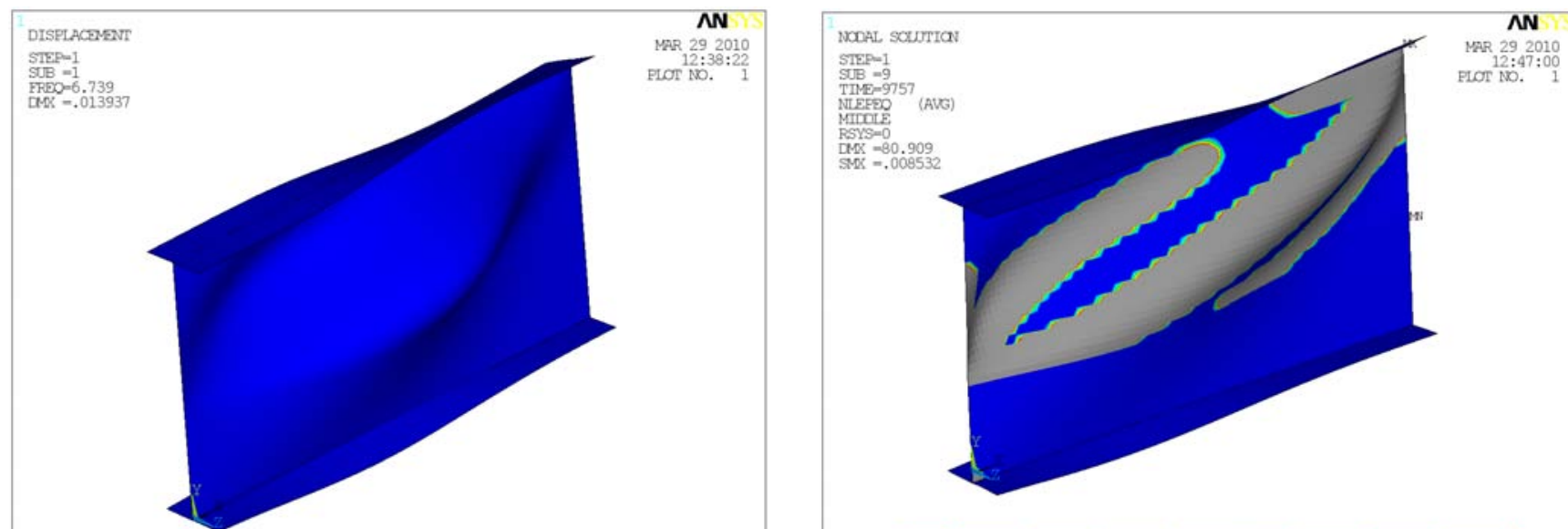

Fig. 5: Bottom $1500 \mathrm{~mm}$ of web with thickness $50 \mathrm{~mm}$. Criticat snear load $6740 \mathrm{kN}(t \mathrm{eft})$. Resistance 9760 $k N$ as determined by nonlinear analysis. 
In all the analysis above, there is no rotational constraint of the upper flange. In a composite bridge structure, the upper flange is attached to the concrete slab via welded studs which offer a rotational constraint of the entire upper flange. Obviously the beneficial effect on resistance is an interesting matter.

For that purpose the analysis is performed by a rotational constraint of the upper flange. The resistances for i) uniform $20 \mathrm{~mm}$ flange ii) $500 \mathrm{~mm}$ iii) $1000 \mathrm{~mm} \&$ iv) $1500 \mathrm{~mm}$ height of thickness $50 \mathrm{~mm}$ increased from i) 6900 to $7000 \mathrm{kN}$, ii) 7650 to $7750 \mathrm{kN}$, iii) $8540 \mathrm{kN}$ to $8640 \mathrm{kN}$, \& iv) 9760 to $10020 \mathrm{kN}$. These are moderate increases - however this is not because there is no such beneficial effect but rather due to that this effect is already accounted for by the torsionally stiff of the 1000 x 50 flange.

Reducing for that purpose the flanges to the moderate $400 \times 20$ results in resistances of i) $6000 \mathrm{kN}$, ii) $6600 \mathrm{kN}$, iii) $7400 \mathrm{kN}$ \& iv) $8070 \mathrm{kN}$, respectively. Hence, it is seen that the influence of the rotational constraints provided by a stiff flange or a composite flange may enhance the shear resistance by an amount of 15-25\%. This is certainly a factor to take into account in bridge design.

Morover, the interaction between shear force and bending moment is important in bridge design. Considering the uniform thickness web $4000 \times 20 \mathrm{~mm}$ with $1000 \times 50$ flanges, the nominal bending capacity of such a cross-section is $M=355 \cdot 10^{-9} \cdot\left(1000 \cdot 50 \cdot 4000+4000^{2} \cdot 20 / 6\right)=89,9 \mathrm{MNm}$.

In figure 6 and figure 7 below, analysis is made for combinations of simultaneous bending and shear for the cross-section with uniform $20 \mathrm{~mm}$ thickness. The same geometrical imperfections as described above have been applied. For zero shear force, the bending resistance is about $95 \%$ of the nominal elastic resistance due normal stress buckling of the compressed zone of the web. The figures report the bending resistance for $0,25,50 \& 75 \%$ of the shear resistance without bending. In addition, analysis is made also for $95 \%$ of the shear resistance.
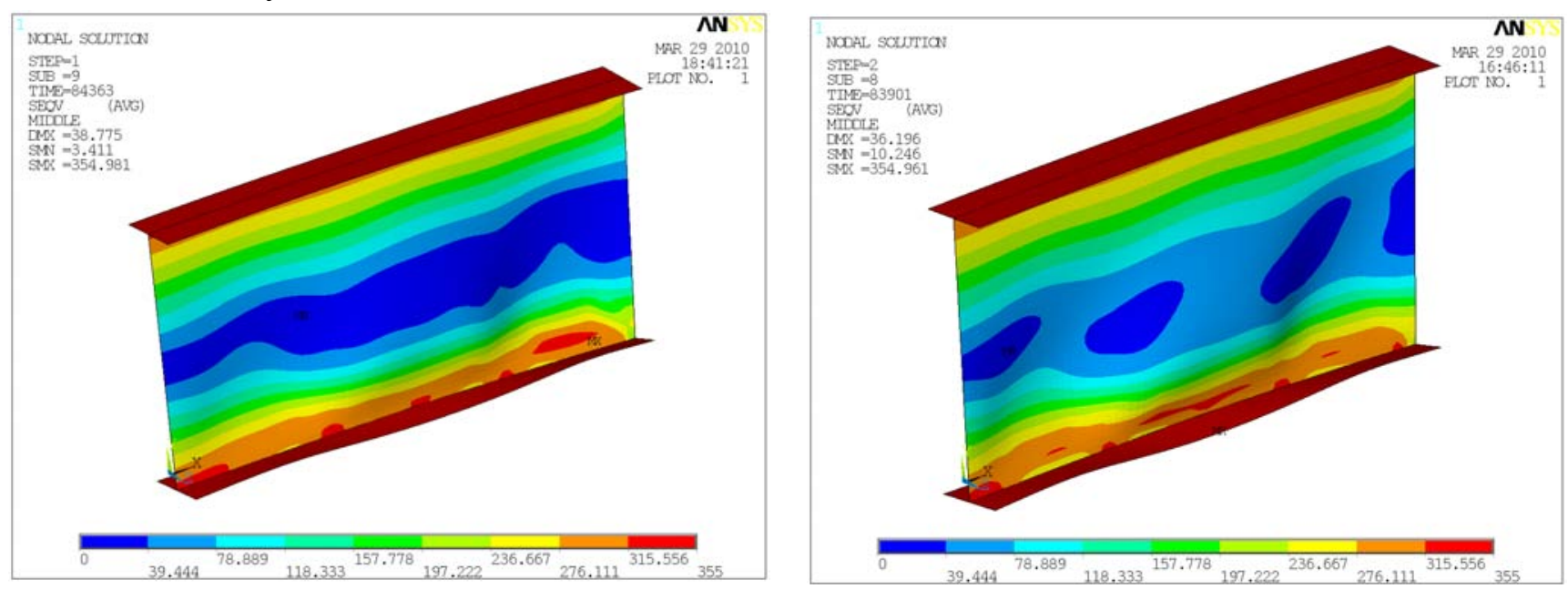

Fig. 6: Uniform thickness web $4000 \times 20 \mathrm{~mm}$. Bending moment resistance 84,4MNm with no shear force (left). Bending moment resistance 83,9 MNm at simultaneous shear force 0,25 x $6900 \mathrm{kN}$ (right).

In figure 8 the corresponding analysis results are found for $0 \& 75 \%$, respectively for the cross section with the bottom $1000 \mathrm{~mm}$ being $50 \mathrm{~mm}$ thick. Analysis is made also for $25,50, \& 95 \%$ but not reported explicitely in figures.

The analysis results are collected into the non-dimensional interaction diagrams as given in figure 9. They are both normated to the resistance of the cross-section with uniform $20 \mathrm{~mm}$ web thickness. This procedure is chosen so as to clearly demonstrate the gains of the thicker bottom web strip. There is some $16 \%$ more in the latter cross-section wheras the resistance gain is $25-30 \%$. Moreover, there is virtually no interaction at all between shear $\&$ bending i.e. the full resistancies may be utilized simultaneously. 

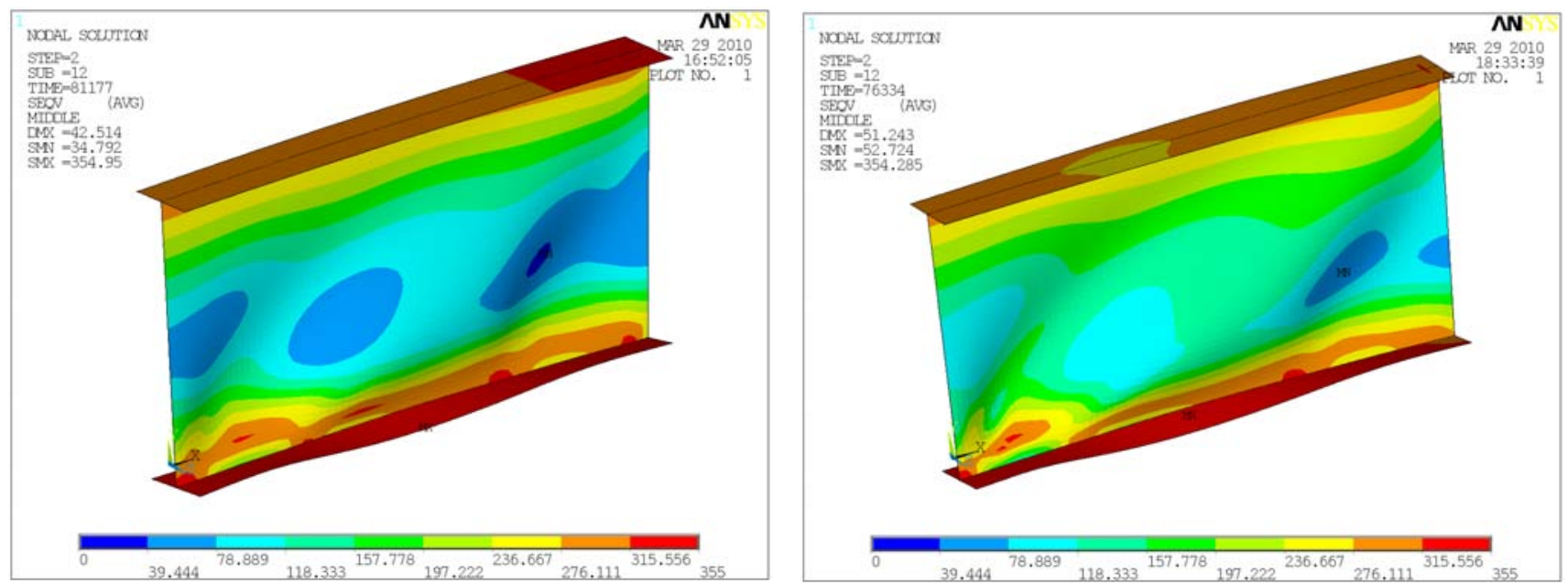

Fig. 7: Uniform thickness web $4000 \times 20 \mathrm{~mm}$. Bending moment resistance 81,2 MNm at simultaneous shear force 0,50 $6900 \mathrm{kN}$ (left). Bending moment resistance 76,3 MNm at simultaneous shear force 0,75 6900 $k N($ right).
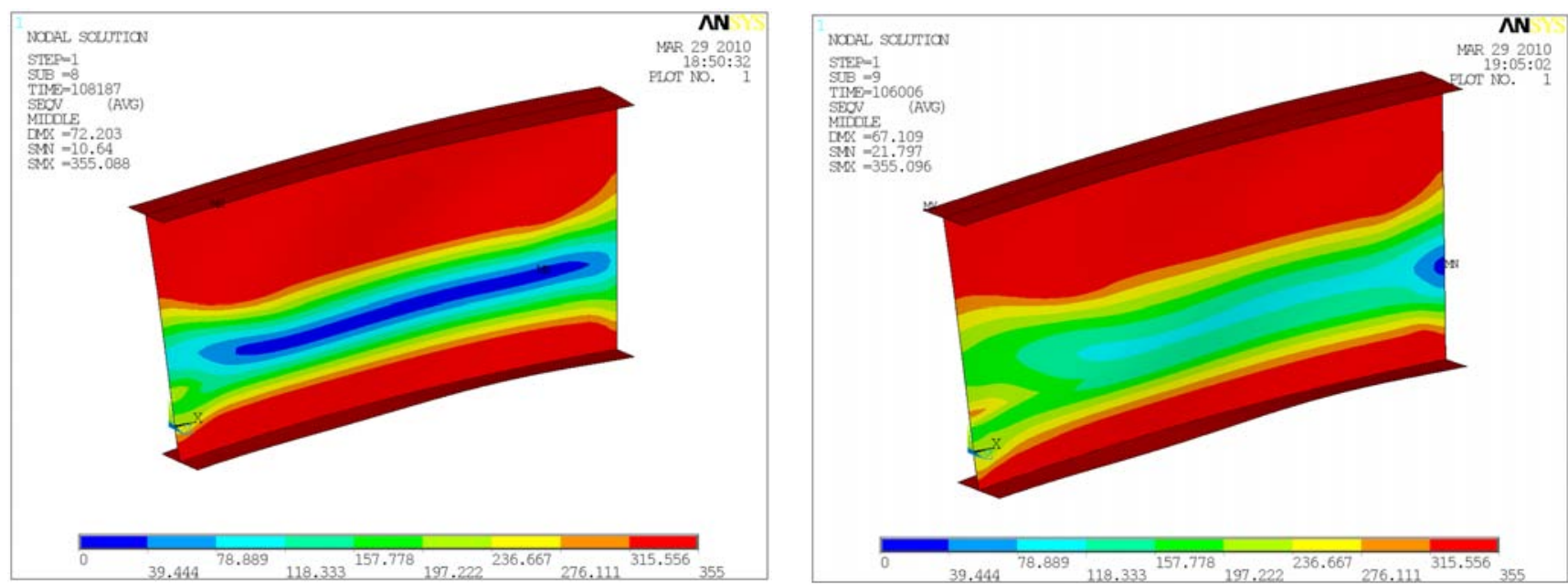

Fig. 8: Bottom $1000 \mathrm{~mm}$ thickness $50 \mathrm{~mm}$. Bending moment resistance 108,2 MNm with no shear force (left). Bending moment resistance 106,0 MNm at simultaneous shear force 0,75 x $8540 \mathrm{kN}$ (right).

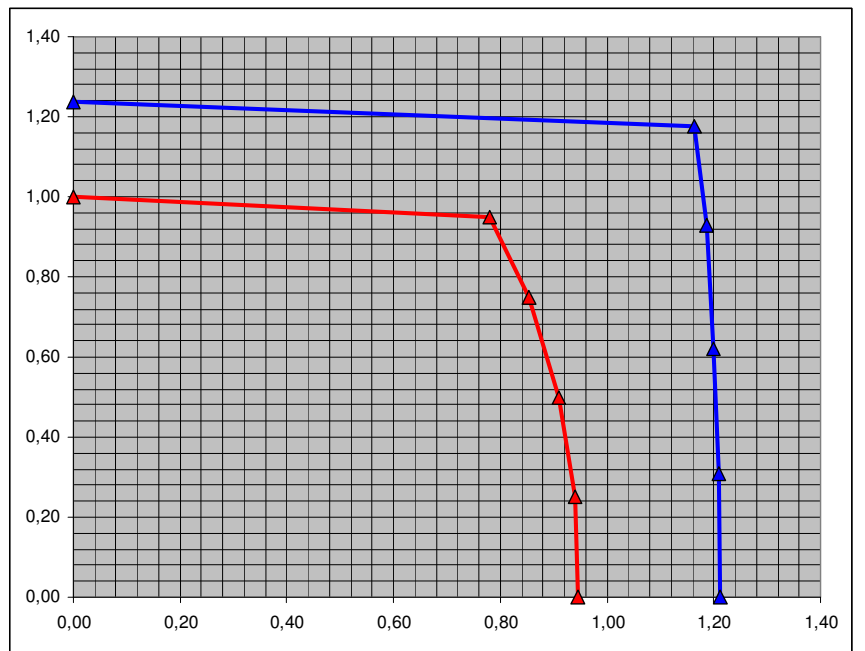

Fig. 9: Interaction curves for bending \& shear. Uniform thickness web $4000 \times 20$ red curve. Bottom 1000 mm web thickness $50 \mathrm{~mm}$ blue curve. Flanges $1000 \times 50 \mathrm{~mm}$. The ordinate is (V/6900) and the abscissa is $(M / 89,3)$ in which $6900 \& 89,3$ are the shear resistance [kN] \& bending resistance [MNm] for the uniform web thickness cross-section. 


\section{Discussions \& conclusions}

For very large girders, the web needs to be manufactured from two plate strips. While there is no remedy to avoid the longitudinal butt weld between the web plates, the bottom strip of the web may be design as an extension of the bottom flange. The thicker web bottom strip increases both the shear \& bending resistance of the cross-section by which longitudinal trapezoidal stiffeners may be eliminated. From the studies above it may be concluded that the considered design exhibits a clear potential in bridge design. Adding some $15 \%$ material without adding any welding improves the resistance by $25-30 \%$. Moreover, it enhances the interaction properties for shear \& bending such that the sensitivity for simultaneous actions is reduced.

\section{References}

[1] Eurocode 3 - Design of Steel structures-Part 1-5: Plated structural elements. EN 1993-1-5. 